\title{
Jamming and Stress Propagation in Particulate Matter
}

\author{
M. E. Cates, J. P. Wittmer \\ Dept. of Physics and Astronomy, University of Edinburgh \\ JCMB King's Buildings, Mayfield Road, Edinburgh EH9 3JZ, GB. \\ J.-P. Bouchaud, P. Claudin \\ Service de Physique de l'Etat Condensé, CEA \\ Ormes des Merisiers, 91191 Gif-sur-Yvette Cedex, France.
}

\begin{abstract}
We present simple models of particulate materials whose mechanical integrity arises from a jamming process. We argue that such media are generically "fragile", that is, they are unable to support certain types of incremental loading without plastic rearrangement. In such models, fragility is naturally linked to the marginal stability of force chain networks (granular skeletons) within the material. Fragile matter exhibits novel mechanical responses that may be relevant to both jammed colloids and cohesionless assemblies of poured, rigid grains.
\end{abstract}

\section{Introduction}

In this paper, we consider the relation between jamming (a kinetic process) and the laws of static mechanical equilibrium of particulate media. We describe first a simple model of jamming in a colloid, sheared between parallel plates. We assume that force chains (linear arrays of rigid particles in contact) develop along the major stress compression axis and span the sample. The resulting jammed state can support a shear stress indefinitely, but does this by virtue of a self-organized internal structure (the force chain array) which has developed in direct response to the applied load itself. If a different load is now applied (e.g. if the material is sheared in some different direction), the force chains cannot sustain the load but must flow and rejam in a different configuration. The model thus provides a simple example of a "fragile" material - one which cannot support certain types of infinitesimal stress increment without plastic reorganisation.

1

Preprint submitted to Elsevier Preprint

28 May 2022 


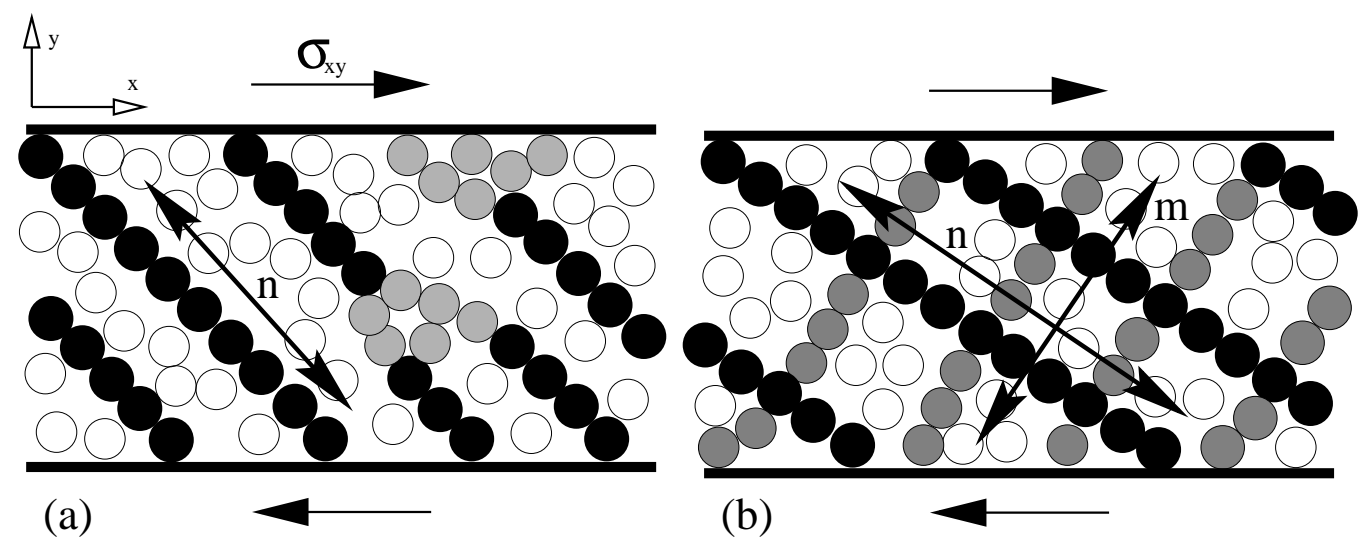

Fig. 1. (a) A jammed colloid (schematic). Black: force chains; grey: other force-bearing particles; white: spectators. (b) Idealized rectangular network of force chains.

The continuum mechanics of fragile materials is very different from conventional elastic or elastoplastic theory. We propose that fragility may be generic in jammed systems, and argue further that a pile of cohesionless poured sand can be viewed as jammed in the required sense: its mechanical integrity results purely from the applied load (gravity). Some simple models that we have recently developed for poured sand [1 44, such as the fixed principal axes (FPA) model, indeed display fragile behaviour; in fact they correspond to assuming a particular simplified geometry for the granular skeleton of force chains.

Fragile models require that the behaviour of a pile of cohesionless grains (fragile) is quite different from a hypothetical pile where each grain is firmly glued to its neighbours on first coming to rest (elastic). This is plausible because for random packings of glued grains, a finite fraction of the interparticle forces will be under tension (forbidden in the cohesionless case) and will remain so until the load is large enough to cause appreciable elastic deformation of individual grains. For simple fragile models such as FPA, the same criterion defines a crossover between fragile behaviour and a form of (anisotropic) elastoplasticity. A fuller discussion of these ideas is given in [1], where further references may be found.

\section{Jamming in Colloids: A Simple Model}

Consider a concentrated colloidal suspension of hard particles, confined between parallel plates at fixed separation, to which a shear stress is applied. Above a certain threshold of stress, this system exhibits enters a regime of strong shear thickening; see, e.g. [5]. The effect can be observed in the kitchen, by stirring a concentrated suspension of corn-starch with a spoon. In fact, computer simulations suggest that, at least under certain idealized conditions, the 


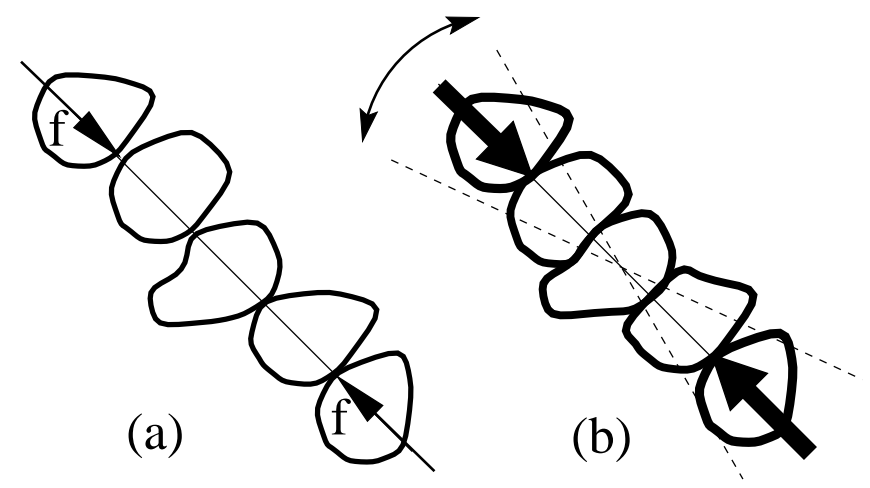

Fig. 2. (a) A force chain of hard particles (any shape) can statically support only longitudinal compression. (b) Finite deformability allows small transverse loads to arise.

material will jam completely and cease to flow, no matter how long the stress is maintained [6]. In these simulations, jamming apparently occurs because the particles form "force chains" [7] along the compressional direction (Fig. 1 (a)). Even for spherical particles the lubrication films cannot prevent direct interparticle contacts; once these arise, an array or network of force chains can indeed support the shear stress indefinitely. (We ignore Brownian motion, here and below, as do the simulations; this could cause the jammed state to have finite lifetime.)

To model the jammed state, we start from a simple idealization of a force chain: a linear string of at least three rigid particles in point contact. Crucially, this chain can only support loads along its own axis (Fig.2(a)): successive contacts must be collinear, with the forces along the line of contacts, to prevent torques on particles within the chain [8]. Note that neither friction at the contacts, nor particle aspherity, can change this "longitudinal force" rule.

As a minimal model of the jammed colloid, we take an assembly of such force chains, characterized by a unique director $\mathbf{n}$, in a sea of "spectator" particles, and incompressible solvent. This is obviously oversimplified, for we ignore completely any collisions between chains, the deflections caused by weak interactions with the spectator particles, and the fact that there must be some spread in the orientation of the chains themselves [6]. With these assumptions, in static equilibrium, with no body forces acting, the pressure tensor $p_{i j}$ (defined as $p_{i j}-\sigma_{i j}$, with $\sigma_{i j}$ the usual stress tensor) must obey

$$
p_{i j}=P \delta_{i j}+\Lambda n_{i} n_{j}
$$

Here $P$ is an isotropic fluid pressure, and $\Lambda(>0)$ a compressive stress carried by the force chains.

Eq. (11) defines a material that is mechanically very unusual. It permits static equilibrium only so long as the applied compression is along $\mathbf{n}$; while this 
remains true, incremental loads (increase or decrease in stresses at fixed major compression of the stress tensor) can be accommodated reversibly, by what is (at the particle contact scale) an elastic mechanism. But the material is certainly not an ordinary elastic body, for if instead one tries to shear the sample in a slightly different direction (causing a rotation of the principal stress axes) static equilibrium cannot be maintained without changing the director $\mathbf{n}$. Now, $\mathbf{n}$ describes the orientation of a set of force chains that pick their ways through a dense sea of spectator particles. Accordingly $\mathbf{n}$ cannot simply rotate; instead, the existing force chains must be abandoned and new ones created with a slightly different orientation. This entails dissipative, plastic, reorganization, as the particles start to move but then re-jam in a configuration that can support the new load.

\section{Jamming and Fragile Matter}

Our model jammed colloid is an idealized example of "fragile matter": it can statically support applied shear stresses (within some range), but only by virtue of a self-organized internal structure, whose mechanical properties have evolved directly in response to the load itself. Its incremental response can be elastic only to compatible loads; incompatible loads (in this case, those of a different compression axis), even if small, will cause finite, plastic reorganizations. The inability to elastically support some infinitesimal loads is our proposed definition of the term "fragile" (which, up to now, has not been given a precise technical meaning in this context).

We argue that jamming may lead generically to mechanical fragility, at least in systems with overdamped internal dynamics. Such a system is likely to arrests as soon as it can support the external load; since the load is only just supported, one expects the state to be only marginally stable. Any incompatible perturbations then force rearrangement; this will leave the system in a newly jammed but (by the same argument) equally fragile state. This scenario is related, but not identical, to several other ideas in the literature 8 13. These include the emergence of rigidity by successive buckling of force chains in glasses and granular matter [9]; the concepts of self-organized criticality (SOC) [10] (see also [3]), and those of mechanical percolation which underly recent "hypoplastic" models of granular matter [1]. Fragility is also connected with recent ideas concerning isostaticity and marginal mechanics in (frictionless) sphere packings [12, 13] (see Section 7).

Our ideas are, in addition, reminiscent of the (much older) concept of a selfselecting critical state in theories of soil mechanics 114. However the latter is primarily concerned with dilatancy: the tendency of dense particulate media to expand upon shearing. Jamming can be viewed as the constant-volume 
counterpart of this process: if expansion is prevented, jamming results.

\section{Two Types of Fragility}

Consider the idealized jammed colloid of (Fig. 1 (a)). So far we allowed for an external stress field (imposed a the plates) but no body forces. What body forces can it now support without plastic rotation of the director? Various models are possible. One is to assume that Eq. (目) continues to apply, with $P(\mathbf{r})$ and $\Lambda(\mathbf{r})$ now varying in space. If $P$ is a simple fluid pressure, a localized body force can be supported only if it acts along $\mathbf{n}$. Thus (as in a bulk fluid) no static Green function exists for a general body force. (Note that, since Eq. (11) is already written as a continuum equation, such a Green function would describes the response to a load that is localized in space but nonetheless acts on many particles in some mesoscopic neighbourhood.)

For example, if the particles in Fig. 11 (a) were to become subject to a gravitational force along $y$, then the existing force chains could not sustain this but would reorganize. Applying the longitudinal force rule, the new shape is easily found to be a catenary, as realized by Hooke [15], and emphasized by Edwards 8]. On the other hand, a general body force can be supported, in three dimensions, if there are several different orientations of force chain, possibly forming a network or "granular skeleton" [7, 11, 17, 18]. A minimal model for this is:

$$
p_{i j}=\Lambda_{1} n_{i} n_{j}+\Lambda_{2} m_{i} m_{j}+\Lambda_{3} l_{i} l_{j}
$$

with $\mathbf{n}, \mathbf{m}, \mathbf{l}$ directors along three nonparallel populations of force chains; the $\Lambda$ 's are compressive pressures acting along these. Body forces cause $\Lambda_{1,2,3}$ to vary in space.

We can thus distinguish two levels of fragility, according to whether incompatible loads include localized body forces (bulk fragility, e.g. Eq. (11)), or are limited to forces acting at the boundary (boundary fragility, e.g. Eq. (2)). In disordered systems one should also distinguish between macro-fragile responses involving changes in the mean orientation of force chains, and the micro-fragile responses of individual contacts. Below we focus on macro-fragility, but in practice the distinction may become blurred. In any case, these various types of fragility should not be associated too strongly with minimal models such as Eqs. (11,21). It is clear that many granular skeletons have a complex network structure where many more than three directions of force chains exist. Such a network may nonetheless be fragile; see Section 7 below. 


\section{Fixed Principal Axis (FPA) Model}

Returning to the simple model of Eq. (2), the chosen values of the three directors (two in 2-d) clearly should depend on how the system came to be jammed (its "construction history"). If it jammed in response to a constant external stress, switched on suddenly at some earlier time, one can argue that the history is specified purely by the stress tensor itself. In this case, if one director points along the major compression axis then by symmetry any others should lie at rightangles to it (Fig. 11 (b)). Applying a similar argument to the intermediate axis leads to the ansatz that all three directors lie along principal stress axes; this is perhaps the simplest model in 3-d. One version of this argument links force chains with the fabric tensor [11], which is then taken coaxial with the stress [18.

With the ansatz of perpendicular directors as just described, Eq. (2) becomes a "fixed principle axes" (FPA) model [1,2]. Although grossly oversimplified, this leads to nontrivial predictions for the jammed state in the colloidal problem, such as a constant ratio of the shear and normal stresses when these are varied in the jammed regime. Such constancy is indeed reported by Laun [5] in "the regime of strong shear thickening"; see [4].

\section{Granular Materials}

We now turn from colloids to granular materials. Although the formation of dry granular aggregates under gravity is not normally described in terms of jamming, it is a closely related process. Indeed, the filling of silos and the motion of a piston in a cylinder of grains both exhibit jamming and stick-slip phenomena associated with force chains; see [19]. And, just as in a jammed colloid, the mechanical integrity of a sandpile disappears as soon as the load (in this case gravity) is removed.

In the granular context, a model like Eq. (2) is interpreted by saying that a fragile granular skeleton of force chains is laid down at the time when particles are first buried at the free surface; so long as subsequent loadings are compatible with this structure, the skeleton will remain intact. If in addition the skeleton is rectilinear (perpendicular directors) this forces the principal axes to maintain forever the orientation they had close to the free surface (FPA model). However, we do not insist on this last property and other models, based on an oblique family of directors, have also been developed [1.,4]. (The construction history of a sandpile allows this since the orientation of the free surface and/or gravity provides a reference direction in addition to that given by the stress tensor at the onset of jamming [1].) Note also that, for a conical 
sandpile we require in addition to Eq. (2) one further relation among stresses, found for example by assuming that $\Lambda_{2}=\Lambda_{3}$ everywhere; see [1]. It turns out that the FPA models account quite well for the forces measured experimentally beneath conical piles of sand, constructed by pouring cohesionless grains from a point source onto a rough rigid support [20,1]. (Note that the two dimensional case - a wedge - may have special features and the effectiveness of FPA for this is much less clear [4].)

As mentioned previously, fragile models such as this show very different mechanics from conventional forms of elasticity or elastoplasticity. For example, in 2-d, when combined with stress continuity $\left(\partial_{i} \sigma_{i j}=\rho g_{j}\right.$ for sand under gravity), Eq. (2) gives differential equations for the stress tensor which are hyperbolic [2]. With a zero-force boundary condition at the upper surface of a pile, this gives a well-posed problem: the forces acting at the base follow uniquely from the body forces by integration. (Analogous remarks apply to Eq. (2) in 3-d.) If different forces are now imposed at the base, rearrangement is inevitable [21]. (This is boundary-fragile behaviour.) The same does not hold [4] within a traditional elastoplastic modelling approach [22] whose equations are elliptic in elastic zones. In such models of the sandpile the forces acting at the base cannot be found without specifying a displacement field there. To define this displacement, one would normally invoke as reference state the one in which the load (gravity) is removed. For cohesionless poured sand, this state is undefined [23], just as it is for a jammed colloid which, in the absence of the applied shear stress, is simply a fluid.

One route to an elastic reference state is to consider a hypothetical sandpile where each grain becomes firmly "glued" to its neighbours (or the base), upon first coming to rest. The resulting medium is surely elastic, and must therefore be governed by elliptic equations. This does not mean that it is a conventional homogeneous elastic continuum (for which the states of zero strain and zero stress coincide). Indeed, a glued pile built under gravity will certainly have nonzero stresses if gravity is later removed «. More importantly, for a typical disordered packing of near-rigid, glued grains, there will arise many tensile contacts even under a purely compressive external load. Thus the problem of glued and unglued piles might, in practice, be extremely dissimilar.

\section{Isostaticity, Marginal Packings, and Fragility}

The problem of cohesionless granular media is a highly nonlinear one, because (a) grains are typically very rigid (on a scale set by the stresses that arise), and (b) there can be no tensile forces between any grains in the entire system. The first issue is addressed in [4] where we show that, for some fragile models, there is in fact a smooth crossover to more conventional elastoplastic physics 
when grains are deformable, with fragility emerging as the limiting behaviour for rigid particles.

In this rigid particle limit, where the longitudinal force rule (LFR) becomes valid, fragility will be recovered in a granular skeleton of force chains whose coordination number $z=2 d$ with $d$ dimension of space (e.g. Fig. 1 (b) in two dimensions). This is the same rule as applies for packings of frictionless hard spheres, which also obey the LFR - not because of force chains, but because there is no friction. Indeed, regular packings of frictionless spheres, which show "marginal" or "isostatic" mechanics have been studied in detail recently [12]. Note that although our idea of fragility is closely related to the concept of isostaticity (see e.g. [13]), it is apparently not identical; in our models of fragile materials the isostatic condition applies only to a loadbearing substructure (the force skeleton) and not the whole packing. It would be interesting to test this idea numerically by measuring the mean coordination number among the subset of particles that carry strong forces.

Interestingly, Moukarzel has recently argued [13 that random packings of frictionless spheres generically become isostatic $(z=2 d)$ in the rigid particle limit. His arguments appear to depend only on the LFR and the absence of tensile forces, so they should, if correct, equally apply to any granular skeleton that is made of force chains of rigid particles. It remains to be seen whether Moukarzel's (somewhat unintuitive) arguments can be made rigorous. Pending this, we prefer for the moment our own qualitative reasoning, which generically links fragility to jamming, as the motivation for setting $z=2 d$ in our simplified models (such as FPA) of the granular skeleton. Such models, in which the skeleton is represented as a rectilinear or oblique array with no disorder, are obviously convenient for calculation. However, disorder will not remove the fragility (though it will change Eqs. (11,2)) unless it causes the mean coordination number of the skeleton to increase.

\section{Conclusions}

The jammed state of colloids, if it indeed exists in the laboratory, has not yet been fully elucidated by experiment. It is interesting the even very simple models such as Eq. (1) can lead to nontrivial and testable predictions (such as the constancy of certain measured stress ratios). Such models suggest an appealing conceptual link between jamming, force chains, and fragile matter [1]. However, further experiments are needed to establish the degree to which they are useful in describing real colloids.

For granular media, the existence of tenuous force-chain skeletons is clear [0,11,16 18]; the question is whether such skeletons are fragile. Several theo- 
retical arguments have been given, above and elsewhere, to suggest that this may be the case, at least in the limit of rigid particles. Moreover, simulations show strong rearrangement under small changes of compression axis; the skeleton is indeed "self-organized" [17, 18]. Experiments also suggest cascades

of rearrangement [3, 19] in response to small disturbances. These findings are consistent with the fragile picture.

The mechanics of fragile models such as Eqs. (11,2) differ strongly from those of conventional elasticity. For example, if an infinitesimal point force is exerted on top of a layer of sand, we expect the resulting pressure distribution at the base to form an annulus [2]; an elastic model would predict the maximum pressure to be directly beneath the applied force. It could be very fruitful to perform new (but quite simple) experiments of this kind.

\section{Acknowledgement}

We thank R. Ball, E. Clement, S. Edwards, M. Evans, P. Evesque, P.-G. de Gennes, G. Gudehus, J. Goddard, J. Jenkins, D. Levine, J. Melrose, S. Nagel, J. Socolar, C. Thornton, L. Vanel and T. A. Witten for discussions. Work funded in part by EPSRC (UK) GR/K56223 and GR/K76733.

\section{References}

[1] J. P. Wittmer, P. Claudin, M. E. Cates and J.-P. Bouchaud, Nature, 382, 336 (1996); J. P. Wittmer, P. Claudin, M. E. Cates, J. Physique I, 7, 39 (1997).

[2] J.-P. Bouchaud, M. E. Cates, and P. Claudin, J. Physique I, 5639 (1995); J.-P. Bouchaud, P. Claudin, M. E. Cates, J. P. Wittmer, in Physics of Dry Granular Media, H.J. Herrmann, J.P. Hovi and S. Luding, Eds., NATO ASI 1997 (cond-mat/9711135).

[3] P. Claudin and J.-P. Bouchaud, Phys. Rev. Lett. 78, 231 (1997); P. Claudin, J.-P. Bouchaud, M. E. Cates and J. P. Wittmer, Phys. Rev. E, 57, 4441 (1998).

[4] M. E. Cates, J. P. Wittmer, J.-P. Bouchaud, P. Claudin, Phys. Rev. Lett., to be published (cond-mat/9803197); Phil. Trans. Roy. Soc. Lond. Ser. A., to be published (cond-mat/9803266).

[5] H. M. Laun, J. Non-Newtonian Fluid Mech., 54, 87 (1994).

[6] R. S. Farr, J. R. Melrose, and R. C. Ball, Phys. Rev. E, 55, 7203 (1997); R. C. Ball, J. R. Melrose, Adv. Colloid Interface Sci., 59, 19 (1995); J. R. Melrose, R. C. Ball, Europhys. Lett., 32, 535 (1995). 
[7] P. Dantu, Annales des Ponts et Chaussées, 4, 144 (1967).

[8] S. F. Edwards and C. C. Mounfield, Physica A 226, 1, 12, 25 (1996); S. F. Edwards, Physica A 249, 226 (1998).

[9] S. Alexander, Phys. Reports 296, 65 (1998); P. S. Cundall and O. D. L. Strack, in Mechanics of Granular Materials: New Models and Constitutive Relations, J.. T. Jenkins and M. Satake, Eds, Elsevier 1983.

[10] P. Bak, How Nature Works; The Science of Self-Organized Criticality (Oxford University Press, Oxford 1997).

[11] D. Kolymbas, Archive of Appl. Mech., 61, 143 (1991); G. Gudehus, in Ref. [22].

[12] R. C. Ball and S. F. Edwards, in preparation; D. V. Grinev and R. C. Ball, in preparation.

[13] C. Moukarzel, preprint cond-mat/9803120); to appear in Proc. Rigidity Theory and Applications, Fundamental Material Science Series, Plenum condmat/9807004).

[14] See, e.g., D. Muir Wood, Soil Behaviour and Critical State Soil Mechanics Cambridge University Press, 1990.

[15] R. Hooke, A description of helioscopes, and some other instruments John Martin, London (1676). See anagram on title page concerning "The true Mathematical and Mechanical form of all manner of Arches."; reproduced as Fig.2.2 of [14.

[16] C. H. Liu, S. R. Nagel, D. A. Schecter, S. N. Coppersmith, S. Majumdar and T. A. Witten, Science 269513 (1995); S. R. Nagel, Rev. Mod. Phys., 64, 321 (1992).

[17] C. Thornton, in KONA Powder and Particle 15 (1997); C. Thornton and G. Sun, in Numerical Methods in Geotechnical Engineering, I. Smith, Ed., Balkema, 143-148 (1994).

[18] F. Radjai, D. E. Wolf, M. Jean, J. J. Moreau, Phys. Rev. Lett. 90, 61 (1998), and references therein.

[19] E. Kolb, T. Mazozi, J. Duran, E. Clement, in preparation. P. Claudin, J.P. Bouchaud, cond-mat/9801057, to appear in Granular Matter.

[20] Smid, J. and Novosad, J. in Proc. of 1981 Powtech Conf., Ind. Chem. Eng. Symp. 63, D3V 1-12 (1981); R. Brockbank, J. M. Huntley and R. C. Ball, J. Physique II, 7, 1521 (1997).

[21] This was first pointed out by P. Evesque (private communication 1996).

[22] See, e.g., Proc. 3rd Int. Conf. on Powders and Grains, Durham NC 18-23 May 1997, R. P. Behringer and J. T. Jenkins, Eds. Balkema, Rotterdam 1997, especially F. Cantelaube and J. D. Goddard, pp. 185-194. 
[23] But see P.-G. de Gennes, Rev. Mod. Phys. in press, P. Evesque and P.-G. de Gennes, Compt. Rend. a l'Acad. des Sci. in press for recent discussions. 